\title{
Doenças tóxicas de bovinos em Mato Grosso do Sul
}

\section{Toxic diseases of bovines from of Mato Grosso do Sul}

\author{
Roosevelt Isaias Carvalho Souza ${ }^{1 *}$; Ariany Carvalho dos Santos ${ }^{1}$; Nickolly \\ Lilge Kawski de Sá Ribas²; Edson Moleta Colodel ${ }^{3}$; Paula Velozo Leal ${ }^{4}$; Rayane \\ Chitolina Pupin ${ }^{4}$; Nilton Marques Carvalho ${ }^{5}$; Ricardo Antônio Amaral de Lemos ${ }^{6}$
}

\section{Resumo}

Foi realizado um estudo retrospectivo de um período de 13 anos, entre 2000 e 2012, nos arquivos do Laboratório de Anatomia Patológica (LAP), da Faculdade de Medicina Veterinária e Zootecnia (FAMEZ), Universidade Federal de Mato Grosso do Sul (UFMS). Para esse estudo utilizaram-se 2.359 laudos de necropsias de bovinos que tinham conclusão diagnóstica. Em 151 deles $(6,40 \%)$ as causas de morte foram atribuídas às doenças tóxicas, agrupadas em intoxicações por plantas e outras doenças tóxicas. As intoxicações por plantas foram responsáveis por $88,9 \%$ dos surtos diagnosticados. Em ordem decrescente de frequência, as intoxicações pelas seguintes plantas foram descritas: Brachiaria spp. $(27,88 \%)$, Vernonia rubricaulis (25\%), Amorimia pubiflora (11,54\%), Senna occidentalis e S. obtusifolia $(8,65 \%)$, Enterolobium contortisiliquum e polpa cítrica (3,85\% cada), Stylosanthes spp. (2,88\%), Tetrapterys multiglandulosa (1,92\%), Manihot spp., Simarouba versicolor, Crotalaria spp., Pterodon emarginatus e Solanum malacoxylon $(0,96 \%$ cada). Neste grupo também foram agrupadas as nefropatias tóxicas, responsáveis por $9,62 \%$ dos surtos. Dentre as outras doenças tóxicas diagnosticadas, são descritas as intoxicações por chumbo $(30,77 \%)$, ureia $(23,08 \%)$, cloreto de sódio, abamectina e acidentes ofídicos (15,38\% cada). Neste estudo, $5,6 \%$ dos diagnósticos conclusivos realizados em bovinos de Mato Grosso do Sul (MS) durante o período estudado foram atribuídos à ingestão de plantas tóxicas, o que evidencia a importância deste diagnóstico e suas perdas econômicas. Os surtos de intoxicação por Brachiaria spp. foram mais frequentes, mas sua importância como planta tóxica é pequena quando comparada à extensão territorial em que está plantada. Entretanto, os casos subnotificados podem modificar a importância da Brachiaria spp. como planta tóxica.

Palavras-chave: Doenças de bovinos, intoxicações por plantas, doenças tóxicas, estudo retrospectivo, epidemiologia, patologia, Mato Grosso do Sul

\footnotetext{
Abstract

A retrospective study has been conducted for a period of 13 years, between 2000 and 2012, on files archived at the Laboratório de Anatomia Patológica (LAP), at the Faculdade de Medicina Veterinária e Zootecnia (FAMEZ) at Universidade Federal de Mato Grosso do Sul (UFMS). For this study, 2.359

1 Discentes de Doutorado do Programa de Pós-Graduação em Ciência Animal, Faculdade de Medicina Veterinária e Zootecnia, FAMEZ, Universidade Federal de Mato Grosso do Sul, UFMS, Campo Grande, MS. E-mail: carvalhomv6@hotmail.com; arianycarvalho.vet@gmail.com

2 Discente de Mestrado do Programa de Pós-Graduação em Ciência Animal, FAMEZ, UFMS, Campo Grande, MS. E-mail: nickollylk@yahoo.com.br

3 Prof. Adjunto, Dept $^{\circ}$ de Clínica Médica Veterinária, Faculdade de Agronomia e Medicina Veterinária, Universidade Federal de Mato Grosso, UFMT, Cuiabá, MT. E-mail:moleta@gmail.com

4 Bolsistas de Iniciação Científica PIBIC-CNPq, FAMEZ/UFMS, Campo Grande, MS. E-mail: paulavleal@hotmail.com; ray_ pupin_@hotmail.com

5 Técnico de Nível Superior, Médico Veterinário, FAMEZ/ UFMS, Campo Grande, MS. E-mail: nmarques.carvalho@gmail.com

6 Prof. Adjunto do Curso de Medicina Veterinária, FAMEZ/UFMS, Campo Grande, MS. E-mail: ricardo.lemos@ufms.br

* Autor para correspondência 
necropsy reports of cattle with conclusive diagnostic were used. On 151 of them $(6.40 \%)$ the causes of death were attributed to toxic diseases that were grouped under the tags: plant poisonings and other poisoning diseases. Plant poisonings were responsible for $88.9 \%$ of the diagnosed outbreaks. In decreasing order of frequency, poisoning from the following plants was described as: Brachiaria spp. (27.88\%), Vernonia rubricaulis (25\%), Amorimia pubiflora (11.54\%), Senna occidentalis and S. obtusifolia (8.65\%), Enterolobium contortisiliquum and citric pulp (3.85\% each), Stylosanthes spp. (2.88\%), Tetrapterys multiglandulosa (1.92\%), Manihot spp., Simarouba versicolor, Crotalaria spp., Pterodon emarginatus and Solanum malacoxylon ( $0.96 \%$ each). In this group, toxic nephropathy was also included, accounting for $9.62 \%$ of the outbreaks. Among other toxic diseases that have been diagnosed, lead poisoning $(30.77 \%)$, urea $(23.08 \%)$, sodium chloride, abamectin, and snakebites $(15.38 \%$ each) are described. In this study, $5.6 \%$ of conclusive diagnoses performed on cattle from Mato Grosso do Sul (MS) during the period studied were attributed to ingestion of toxic plants, which highlights the importance of this diagnostic and their economic losses. Outbreaks of Brachiaria spp. were more frequent, but its importance as a toxic plant is small when compared to the extension of the fields on which it is planted. However, the underreported cases can change the importance of Brachiaria spp. as a toxic plant.

Key words: Bovine diseases, plant poisonings, toxic diseases, retrospective study, epidemiology, pathology, Mato Grosso do Sul

\section{Introdução}

As doenças tóxicas que acometem bovinos são responsáveis por importantes perdas econômicas em Mato Grosso do Sul (MS). Embora a importância das intoxicações por planta possa variar de região para região, muitas plantas tóxicas causam prejuízos consideráveis em várias regiões em virtude da existência dessas espécies difundidas no Brasil (TOKARNIA et al., 2012). Desta maneira, a maioria das intoxicações por plantas no MS ocorre em outras regiões brasileiras. Até o momento tem sido descritos trabalhos referindo-se a relatos de surtos de intoxicações por Vernonia rubricaulis (BRUM et al., 2002), Tetrapterys multiglandulosa (CARVALHO et al., 2006), Brachiaria spp. (SOUZA et al., 2010), Stryphnodendron fissuratum (FERREIRA et al., 2009), Simarouba versicolor (CARVALHO et al., 2013), Senna occidentalis e Senna obtusifolia (FERREIRA-NETO et al., 2004, SANTOS et al., 2011) e outros agentes como intoxicação por polpa cítrica (SPLENGLER et al., 2006) e intoxicação por chumbo (LEMOS et al., 2004) em MS. No entanto, não há estudos retrospectivos comparativos sobre a prevalência de doenças tóxicas em bovinos no Estado.

Estudos retrospectivos baseados na coleta de dados em arquivos são cada vez mais importantes na Patologia Veterinária, pois é através destes levantamentos que se pode definir a prevalência de uma doença em uma região, agrupar os dados epidemiológicos, clínicos e patológicos, além de possibilitar a utilização de técnicas complementares de diagnóstico para caracterizar a etiologia das lesões anteriormente observadas e de causa indeterminada, permitindo modificar diagnósticos incorretos ou conceitos errôneos (FIGHERA, 2008).

O objetivo desse trabalho foi descrever a frequência e os aspectos epidemiológicos e patológicos das doenças tóxicas que afetam o rebanho bovino Sul Mato-grossense, por meio de estudo retrospectivo dos diagnósticos realizados no Laboratório de Anatomia Patológica (LAP), da Faculdade de Medicina Veterinária e Zootecnia (FAMEZ), Universidade Federal de Mato Grosso do Sul (UFMS), no período de 2000 a 2012.

\section{Material e Métodos}

Realizou-se um estudo sobre a frequência das doenças tóxicas nos arquivos do LAP/FAMEZ, utilizando-se os laudos de necropsias e exames histopatológicos da espécie bovina, emitidos entre os meses de janeiro de 2000 e dezembro de 2012. Os dados foram obtidos em necropsias 
realizadas por médicos veterinários do LAP ou por profissionais autônomos que enviaram material para o exame histopatológico. Os casos experimentais, os materiais provenientes de outros estados e os diagnósticos inconclusivos foram excluídos desse estudo.

Foram consideradas doenças tóxicas aquelas causadas pela intoxicação por plantas, medicamentos, resíduo industrial de fruto cítrico, metais pesados, sais e acidentes ofídicos. Foram classificados como nefropatias tóxicas os casos em que havia fortes evidências de consumo de plantas tóxicas e outras causas de nefropatia foram descartadas. Desta forma, as doenças tóxicas dos bovinos foram agrupadas em: intoxicações por plantas (ação tóxica ou mecânica) e outras doenças tóxicas. A intoxicação por polpa cítrica foi categorizada dentro das intoxicações por plantas de acordo com Tokarnia et al. (2012).

Por meio da revisão dos laudos, o levantamento foi distribuído por número de casos e número de surtos, considerando como surto quando um ou mais materiais de uma mesma propriedade foram remetidos ao LAP/FAMEZ, de acordo com Thrusfield (2004). Dos laudos de necropsia foram obtidos dados epidemiológicos (idade, procedência, época de ocorrência, morbidade e letalidade), sinais clínicos e achados patológicos. Os diagnósticos definitivos de intoxicações por plantas foram baseados na epidemiologia, sinais clínicos e achados patológicos associados à presença da planta na pastagem com evidência de ingestão da mesma pelos bovinos afetados. Para os casos de intoxicação por chumbo foi realizada a dosagem de chumbo em fragmentos de fígado e rim.

Os coeficientes de morbidade e letalidade foram calculados com base na população sob risco, sendo esta considerada como o número de bovinos no lote problema.

\section{Resultados e Discussão}

De janeiro de 2000 a dezembro de 2012, o LAP/ FAMEZ tem 8.535 laudos patológicos arquivados relativos às diversas espécies animais de interesse veterinário. Desses, 5.456 (64\%) referem-se à espécie bovina, porém foram excluídos 3.097 $(56,76 \%)$ diagnósticos por se tratarem de materiais oriundos de outros estados, de casos experimentais ou de diagnósticos inconclusivos. Dos 2.359 $(43,24 \%)$ diagnósticos conclusivos, as doenças tóxicas corresponderam a $151(6,4 \%)$ diagnósticos.

Os dados descritos para cada doença são pontuais, ou seja, foram coletados no momento da requisição dos exames, podendo não corresponder ao total de bovinos afetados em um surto. Nestes surtos o número de bovinos afetados foi, na maioria das vezes, maior que o número de necropsias realizadas. Na Tabela 1 está sumarizada a relação completa das doenças tóxicas (intoxicações por plantas e outras doenças tóxicas), distribuídas em número de casos e surtos, com a respectiva porcentagem, a idade dos bovinos afetados e os coeficientes de morbidade e letalidade.

\section{Intoxicações por plantas}

As intoxicações por plantas constituem importantes causas de mortalidade em bovinos em Mato Grosso do Sul, representando aproximadamente $\quad 5,6 \%$ dos diagnósticos conclusivos realizados durante o período estudado. O percentual obtido neste estudo é menor do que o observado por outros laboratórios de diagnóstico no Brasil. No Rio Grande do Sul, casos de intoxicações por plantas representam, aproximadamente, 7\% a $16 \%$ dos diagnósticos de morte em bovinos (RIET-CORREA; MEDEIROS, 2001; PEDROSO et al., 2007; RISSI et al., 2007). Na Paraíba, esse índice atinge 7,4\% (ASSIS et al., 2010) e, em Santa Catarina, aproximadamente 14\% (RIETCORREA; MEDEIROS, 2001). Os fatores que 
podem ter contribuído para isso estão relacionados ao elevado número de materiais encaminhados com diagnósticos inconclusivos, devido principalmente ao envio de históricos clínicos e dados epidemiológicos incompletos ou ausentes, ou a subnotificação dos casos por produtores e médicos veterinários autônomos. Assim, é provável que o número de casos de intoxicação por plantas no estado seja ainda maior. Considerando que a população de bovinos de Mato Grosso do Sul, estimada em 21 milhões de cabeças, é superior a dos estados acima mencionados (BRASIL, 2012), o total de bovinos acometidos é maior que o observado nestas regiões. Considerando que no Brasil a estimativa de mortalidade anual de bovinos é de aproximadamente 5\% (CORRÊA et al., 2000), a perda de bovinos em Mato Grosso do Sul relacionada às plantas tóxicas pode ser estimada em 60 mil cabeças por ano.

Tabela 1. Doenças Tóxicas diagnosticadas em bovinos pelo Laboratório de Anatomia Patológica (LAP), da Faculdade de Medicina Veterinária e Zootecnia (FAMEZ), Universidade Federal de Mato Grosso do Sul (UFMS), durante o período de 2000-2012.

\begin{tabular}{|c|c|c|c|c|c|c|}
\hline \multicolumn{7}{|c|}{ Doenças tóxicas de Bovinos } \\
\hline Intoxicações por plantas & $\begin{array}{c}\mathrm{N}^{\mathbf{0}} \\
\operatorname{casos}\end{array}$ & $\begin{array}{c}\mathrm{N}^{\circ} \\
\text { surtos }\end{array}$ & $\begin{array}{l}\% \text { dos } \\
\text { surtos }\end{array}$ & $\begin{array}{c}\text { Idade } \\
\text { (meses) }\end{array}$ & $\begin{array}{l}\text { Morbidade } \\
(\%)\end{array}$ & Letalidade (\%) \\
\hline Brachiaria spp. & 29 & 29 & 27,88 & $6-144$ & $0,03-37,5$ & $10-100$ \\
\hline Vernonia rubricaulis & 30 & 26 & 25,0 & $14-60$ & $0,25-100$ & $76,92-100$ \\
\hline Amorimia pubiflora & 17 & 12 & 11,54 & $12-96$ & $0,05-6,55$ & $24-100$ \\
\hline Nefropatia Tóxica & 13 & 10 & 9,62 & $2-36$ & $0,08-100$ & $10-100$ \\
\hline $\begin{array}{l}\text { Senna occidentalis e/ou } S \text {. } \\
\text { obtusifolia }\end{array}$ & 18 & 9 & 8,65 & $12-36$ & $0,17-3,88$ & $11,11-100$ \\
\hline Enterolobium contortisiliquum & 4 & 4 & 3,85 & $18-60$ & $2,06-3,53$ & $40-83,33$ \\
\hline Polpa Cítrica & 5 & 4 & 3,85 & $18-24$ & $2,69-4,26$ & $71,43-100$ \\
\hline Stylosanthes spp. & 3 & 3 & 2,88 & $15-26$ & 0,02 & 100 \\
\hline Tetrapterys multiglandulosa & 5 & 2 & 1,92 & $24-36$ & $3,16-79,31$ & $3,04-100$ \\
\hline Simarouba versicolor & 2 & 1 & 0,96 & 36 & 2,85 & 94,74 \\
\hline Crotalaria spp. & 1 & 1 & 0,96 & 30 & 0,09 & 100 \\
\hline Pterodon emarginatus & 1 & 1 & 0,96 & $>24$ & $\mathrm{NI}^{\mathrm{a}}$ & NI \\
\hline Solanum malacoxylon & 1 & 1 & 0,96 & NI & 1,67 & 2 \\
\hline Manihot spp. & 3 & 1 & 0,96 & $24-36$ & 100 & 100 \\
\hline TOTAL & 132 & 104 & 100,00 & & & \\
\hline Outras doenças tóxicas & $\begin{array}{c}\mathrm{N}^{\circ} \\
\text { casos }\end{array}$ & $\begin{array}{c}\mathrm{N}^{\circ} \\
\text { surtos }\end{array}$ & $\begin{array}{l}\text { \% dos } \\
\text { surtos }\end{array}$ & $\begin{array}{c}\text { Idade } \\
\text { (meses) }\end{array}$ & $\begin{array}{c}\text { Morbidade } \\
(\%)\end{array}$ & Letalidade (\%) \\
\hline Chumbo & 10 & 4 & 30,77 & $24-36$ & $0,06-16,31$ & $50-100$ \\
\hline Ureia & 3 & 3 & 23,08 & 36 & $0,6-10$ & 100 \\
\hline Cloreto de Sódio & 2 & 2 & 15,38 & $15-24$ & NI & 100 \\
\hline Acidente Ofídico & 2 & 2 & 15,38 & $36-150$ & 1,25 & 100 \\
\hline Abamectina & 2 & 2 & 15,38 & $10-24$ & $5,4-14,06$ & $55,56-93,33$ \\
\hline TOTAL & 19 & 13 & 100,00 & & & \\
\hline
\end{tabular}

aNI - Não Informado.

Fonte: Elaboração dos autores. 
As intoxicações por plantas tóxicas anteriores (LEMOS et al., 2009) não foi relatada diagnosticadas no estado foram por Brachiaria spp., Vernonia rubricaulis, Amorimia pubiflora, Senna occidentalis e S. obtusifolia, Enterolobium contortisiliquum, polpa cítrica, Stylosanthes spp., Tetrapterys multiglandulosa, Manihot spp., Simarouba versicolor, Crotalaria spp., Pterodon emarginatus e Solanum malacoxylon (Figura 1). É importante destacar o surto de intoxicação por Simarouba versicolor, planta que em levantamentos

como tóxica para bovinos. Este dado evidencia que, para estimativa adequada da ocorrência das intoxicações por plantas, os levantamentos em séries históricas devem abranger no mínimo dez anos, como os realizados por outros autores (RISSI et al., 2007; PEDROSO et al., 2007), nos quais algumas intoxicações apresentaram alta frequência com ocorrência constante, enquanto outras foram diagnosticadas esporadicamente.

Figura 1. Plantas tóxicas de interesse pecuário em Mato Grosso do Sul: (A) Inflorescência e sementes de Vernonia rubricaulis; (B) Inflorescência de Amorimia pubiflora; (C) Frutos de Enterolobium contortisiliquum; (D) Broto de Simarouba versicolor; (E) Infrutescência de Tetrapterys multiglandulosa e (F) Folhas e frutos de Pterodon emarginatus.

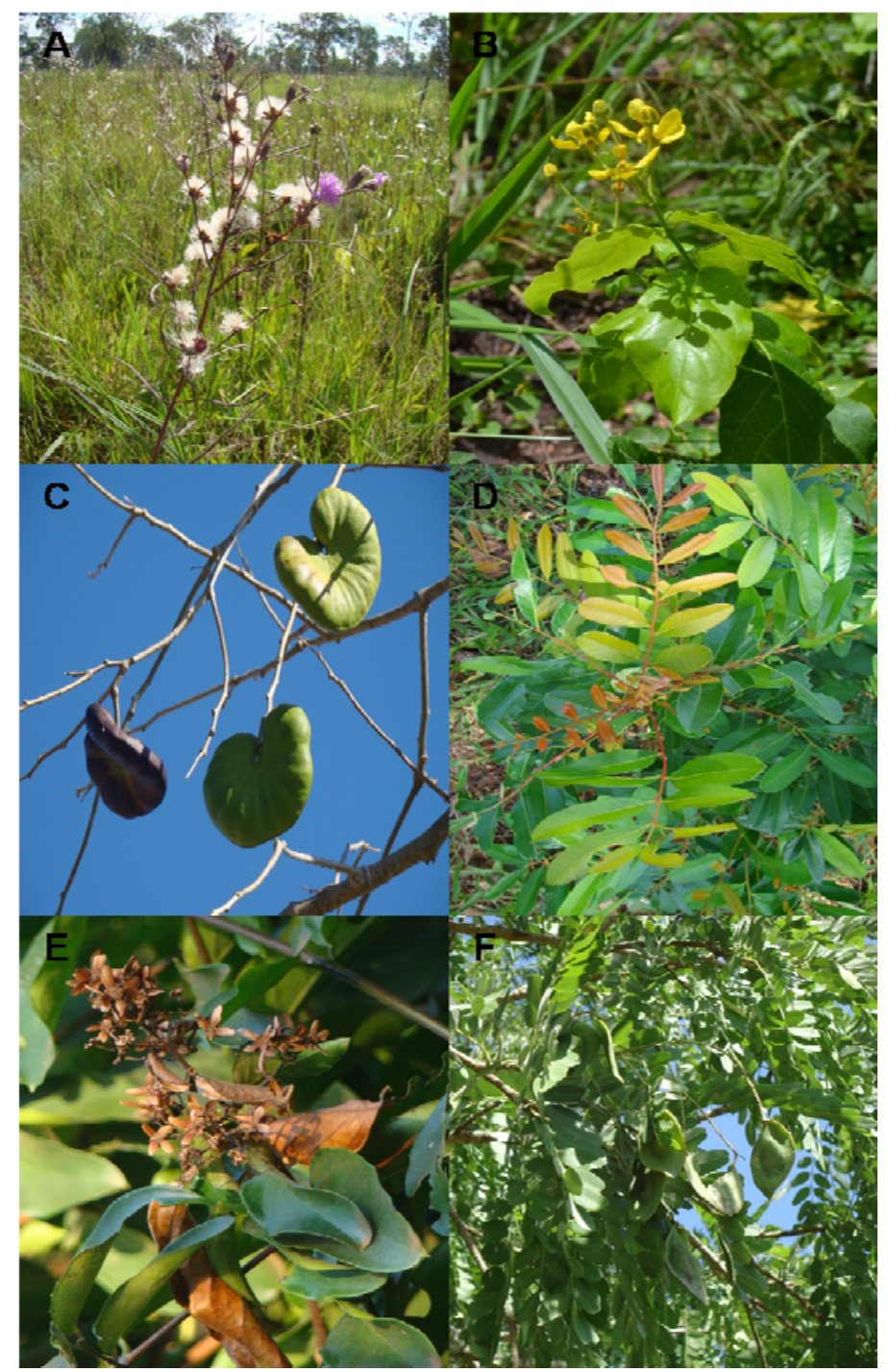

Fonte: Elaboração dos autores. 
Dentre os casos de intoxicações por plantas em bovinos diagnosticados no LAP/FAMEZ, 27,88\% (29 surtos), foram atribuídos à intoxicação por Brachiaria spp. Parte dos surtos de intoxicação por Brachiaria spp. foram descritos pela equipe do LAP/FAMEZ em levantamento anterior (SOUZA et al., 2010), no qual os autores relatam maior ocorrência de casos em bovinos que pastoreiam na $B$. decumbens que $B$. brizantha e que a intoxicação ocorre, principalmente, em bovinos jovens nas diferentes épocas do ano. Estas características mantiveram-se presentes nos demais surtos diagnosticados no LAP/FAMEZ durante os anos seguintes. Esta forrageira, que constitui grande parte das pastagens no Brasil (TOKARNIA et al., 2012) e que nesse estudo afetou bovinos de todas as idades, em todos os meses no ano, com maior prevalência nos meses de julho a agosto, deve ser considerada como uma das principais causas de intoxicação no estado. É provável que esta incidência seja ainda maior, já que vários surtos são subnotificados por produtores ou veterinários, sem o envio de material para exame, ou com a recuperação dos bovinos afetados. A grande variação nos coeficientes de morbidade e letalidade pode estar relacionada à variação da toxicidade entre as diferentes espécies de forrageira, da concentração de saponinas na planta e diferenças da susceptibilidade e resistência entre bovinos de diferentes idades e raças (SOUZA et al., 2010). As plantas do gênero Brachiaria são responsáveis pela fotossensibilização hepatógena, que é induzida por saponinas litogênicas presentes na planta (CRUZ et al., 2000, 2001; BRUM et al., 2007; CASTRO et al., 2009), e também por casos de emagrecimento progressivo (RIET-CORREA et al., 2002; SOUZA et al., 2010).

Vernonia rubricaulis foi incriminada como causa de mortalidade de bovinos em 26 surtos diagnosticados no LAP/FAMEZ, o que correspondeu a $25 \%$ das intoxicações por plantas tóxicas diagnosticadas durante o período estudado. Em todos os surtos desta intoxicação, a ocorrência esteve relacionada a queimadas, passagem de roçadeira na pastagem ou "trilho" (equipamento utilizado para o controle de plantas invasoras, constituído por três barras de trilho de estrada de ferro, puxado por um trator). Estes fatores foram importantes na epidemiologia da intoxicação, uma vez que a planta em brotação é consideravelmente mais tóxica (BRUM et al., 2002). Necrose hepatocelular massiva associada à acentuada congestão e hemorragias centrolobulares, foi o principal achado morfológico em bovinos nesse estudo, semelhantes aos observados por outros autores em mortalidades descritas em bovinos de Mato Grosso do Sul (TOKARNIA; DÖBEREINER, 1982). Destaca-se que apenas um surto foi detalhado anteriormente por Brum et al. (2002) e que outros 25 ocorreram posteriormente durante o período de dez anos. No período estudado a intoxicação pela planta foi diagnosticada em todos os anos e na maioria dos surtos os coeficientes de morbidade foram em torno de $0,25-100 \%$ e letalidade próxima a 100\%.

Amorimia pubiflora foi diagnosticada em 12 surtos (11,54\%) e nos meses de fevereiro, julho, agosto e novembro não houve casos diagnosticados. Os proprietários e responsáveis pelos animais relataram que as mortes ocorreram após a movimentação dos bovinos no mangueiro ou durante o embarque, esta condição é descrita em outros casos espontâneos de intoxicação por $A$. pubiflora em bovinos (BECKER et al., 2013). Os surtos ocorreram independentes da disponibilidade de forragem, no entanto, relata-se frequentemente que as mortes se concentram principalmente no final do período da seca e início do período chuvoso, o que coincide com a fase de brotação, período vegetativo de maior toxidez da planta (TOKARNIA et al., 2012). Em trabalho realizado por Pavarini et al. (2011) no Rio Grande do Sul, nos surtos de intoxicação por Amorimia exotropica (Malpighiaceae), planta que também cursa com morte súbita, os casos ocorreram durante todo o ano, com maior concentração nos meses de maio e agosto, o que os autores relacionam à carência de alimento nesta época do ano no Sul do Brasil. 
Não se determinou a causa de nefropatias tóxicas em dez ocorrências registradas no LAP/FAMEZ. Em dois destes surtos, as lesões histológicas foram compatíveis com intoxicação por oxalato, nas quais foi observada nefrose tubular associada a cristais de oxalato, que são caracterizados por feixes irradiantes birrefringentes (MCGAVIN; ZACARY, 2009). Sendo que um desses, os bovinos pastoreavam Brachiaria humidicola, que tem sido descrita no Brasil como uma das principais plantas que contêm oxalato (TOKARNIA et al., 2012), e no outro que ocorreu em bovinos confinados, Amaranthus spp. era processado junto ao milheto para produção de silagem fornecida aos bovinos, porém, não se reproduziram, experimentalmente, as lesões renais em experimentos realizados no LAP/FAMEZ com uso de silagem coletada in loco. Deve-se considerar que várias tentativas de reprodução da intoxicação com plantas do gênero Amaranthus em locais de surtos também apresentaram resultados negativos (PEIXOTO et al., 2003), ou reproduziram apenas lesões histológicas discretas sem manifestações clínicas (FERREIRA et al., 1991). Isto pode estar relacionado à perda da ação nefrotóxica quando a planta é dessecada ou estocada, como descrita por outros autores em experimentos realizados com Amaranthus spp. (OSWEILLER; BUCK; BICKNELL, 1969; FERREIRA et al., 1991), entretanto, na literatura consultada não há menção se isso também poderia ocorrer no processo de silagem.

Plantas do gênero Senna (S. occidentalis e S. obtusifolia) foram responsáveis por $8,65 \%$ das intoxicações diagnosticadas, sendo 18 casos distribuídos em nove surtos. Os surtos ocorreram em pastejo extensivo e rotacionado em áreas intensamente invadidas pelas plantas. No surto ocorrido em pastoreio rotacionado, um lote de bovinos em terminação foi inicialmente colocado em uma área de milheto, no qual consumiram predominantemente a forrageira, de maneira que quando o lote seguinte entrou predominava $S$. occidentalis em abundância em fase de frutificação, que foi consumida pelos bovinos. Este surto foi descrito detalhadamente por Ferreira-Neto et al. (2004). Nos demais surtos, as áreas estavam intensamente invadidas por $S$. occidentalis ou $S$. obtusifolia ou pelas duas plantas simultaneamente; embora houvesse boa disponibilidade de forragem, a oferta de pastagem não foi avaliada por critérios técnicos, como a taxa de lotação e densidade de pastagem compatíveis com a capacidade de suporte. A ocorrência de surtos em pastoreio semelhante foi descrita anteriormente em surtos no Brasil (BARROS et al., 1999; TAKEUTI et al., 2011; CARMO et al., 2011).

O coeficiente de morbidade, quando considerada apenas a população sob risco, ou seja, o lote de bovinos que foi introduzido posteriormente ao consumo de milheto pelo primeiro lote, foi de 17,6\% (FERREIRA-NETO et al., 2004) o qual é consideravelmente superior aos surtos observados em pastoreio extensivo no presente estudo. O elevado coeficiente de morbidade observado no surto em pastoreio rotacionado evidencia que o aumento na taxa de lotação é um dos fatores de risco para que ocorra a intoxicação por plantas do gênero Senna. Em surto de intoxicação por S. obtusifolia em bovinos em confinamento, descrito por Queiroz et al. (2012) no noroeste do Paraná, o coeficiente de morbidade foi de $10 \%$. Variações nos coeficientes de morbidade, entre $4,2 \%$ a $55,2 \%$, foram observadas em estudo de 16 surtos de intoxicação por $S$. occidentalis em bovinos em pastoreio no Rio Grande do Sul (CARMO et al., 2011). Essas intoxicações ocorreram entre os meses de janeiro e julho, com maior número de casos entre janeiro e março, diferentemente do observado em estudo realizado com 16 surtos de intoxicação no Rio Grande do Sul, onde os casos ocorreram durante o outono e inverno, com 50\% dos surtos em maio (CARMO et al., 2011). Isso poderia estar relacionado a pastos mais pobres com pouca disponibilidade de pastagem naquele estado (HENSON et al., 1965; PIERCE; O'HARA, 1967). O diagnóstico de intoxicação por Senna spp. registrado no LAP/FAMEZ elevou-se 
nos últimos dois anos devido ao aumento destas invasoras na pastagem. Acredita-se que, no presente estudo, o elevado índice pluviométrico que ocorreu na época dos surtos favoreceu o crescimento das plantas do gênero Senna que se sobressaíram às forrageiras. Ressalta-se que a maior invasão ocorreu em pastos de Brachiaria brizantha devido às características vegetativas desta gramínea, que possui hábito de crescimento cespitoso (NUNES et al., 1985), deixando áreas descobertas ao redor, o que favoreceu a germinação das sementes de plantas invasoras, incluindo as do gênero Senna que estavam dormentes no solo. Outro fator que pode estar relacionado ao aumento do número dos casos de intoxicação por Senna spp. foi a vigilância ativa do LAP nas propriedades para o estudo das miopatias tóxicas em bovinos induzidas pelas plantas. Esta condição também é descrita em outros levantamentos (RISSI et al., 2007; SOUTO et al., 2006a; 2006b).

A intoxicação por Enterolobium contortisiliquum representou 3,85\% (quatro surtos) das mortes diagnosticadas dentre as intoxicações por plantas. Os surtos ocorreram entre os meses de julho e setembro, época que coincide com a maturação e queda das favas. Em dois surtos, havia histórico de aborto e fotossensibilização associados à ingestão das favas, achados também descritos por Grecco et al. (2002) e Mendonça et al. (2009). Apesar de E. contortisiliquum ser considerado como causador de fotossensibilização e aborto, a planta também já foi mencionada como causadora de distúrbios digestórios agudos (RIET-CORREA; MEDEIROS; DANTAS, 2006). O diagnóstico dos surtos descritos no presente estudo foi baseado no histórico, nos sinais clínicos e nas lesões macro e microscópicas (GRECCO et al., 2002; MENDONÇA et al., 2009).

Intoxicação por polpa cítrica ocorreu em bovinos confinados (quatro surtos), que recebiam aproximadamente $3,7 \mathrm{~kg}$ do resíduo industrial de frutos cítricos. A ingestão de quantidades da polpa cítrica, iguais ou superiores a $3 \mathrm{~kg}$ diários, por períodos de dois meses ou mais, é associada à intoxicação, sobretudo, em vacas leiteiras (TOKARNIA et al., 2012). Os achados de necropsia se caracterizaram por hemorragias nas serosas, mucosas e tecido subcutâneo nos casos agudos da intoxicação e, à histopatologia, observou-se inflamação granulomatosa com células gigantes no fígado e rim, achado semelhante ao descrito por outros autores (JABOUR et al., 2005; VIEIRA et al., 2007). Detalhes de um dos surtos descritos neste levantamento podem ser obtidos em trabalho realizado por Splengler et al. (2006).

Houve três surtos de obstruções por fitobenzoares de Stylosanthes spp. em bovinos mantidos em pastagem de $B$. brizantha consorciada a estilosantes. Nos três surtos, não foram encontradas lesões histológicas significativas, porém como os materiais foram encaminhados por veterinários autônomos é provável o não encaminhamento do fragmento do intestino correspondente ao local da lesão. Portanto, o diagnóstico foi baseado no envio dos fitobenzoares pelos veterinários, no histórico de falha na consorciação entre o Stylosanthes spp. e a $B$. brizantha e na descrição de necropsia. Segundo notas técnicas do Centro Nacional de Pesquisa em Gado de Corte (CNPGC/Embrapa), que incentivam a consorciação do Estilosantes cultivar Campo Grande ${ }^{\circledR}$, a consorciação máxima não deve ultrapassar 40\% de Stylosanthes spp. em relação a outras gramíneas (EMBRAPA,2007, 2010). Nos três surtos acompanhados, foram relatadas falhas no consórcio entre a leguminosa e pastagem, nos quais a proporção atingiu $70 \%$ de estilosantes em relação a $B$. brizantha, e pode ter favorecido a formação dos fitobenzoares. Ubiali et al. (2013) descreveram, na área de obstrução, necrose da parede intestinal intensamente demarcada em relação a segmento adjacente normal, além de intenso espessamento associado a edema, congestão e hemorragia.

Intoxicações por Tetrapterys multiglandulosa, Simarouba versicolor e Pterodon emarginatus corresponderam, respectivamente, a $1,92 \%, 0,96 \%$ e $0,96 \%$ dos surtos causados por plantas tóxicas. Esses surtos foram descritos em trabalhos realizados 
pelo LAP/FAMEZ (CARVALHO et al., 2006, 2013) e em parceria com a equipe do Laboratório de Patologia Veterinária da Universidade Federal de Mato Grosso (LPV-UFMT) (CRUZ et al., 2012). A intoxicação pelas folhas de $S$. versicolor em bovinos não foi descrita nos anos anteriores. Esta planta ocorre principalmente no cerrado brasileiro e na caatinga, é conhecida como "pau-paraíba", "matacachorro" ou "simarouba-do-Brasil" e, pode atingir três a quatro metros de altura. Neste surto a pastagem foi roçada, o que propiciou a brotação da planta, que se encontrava com cerca de um metro de altura e foi pastejada pelos bovinos. Os sinais clínicos mais comuns foram fraqueza, tremores musculares, incoordenação dos membros pélvicos, decúbito e morte. As lesões histológicas são caracterizadas por necrose do tecido linfoide e enterite necrosante. Experimentalmente, a intoxicação já foi reproduzida em bovinos (CARVALHO et al., 2013) e ovinos (SANTOS et al., 2013).

Um surto de intoxicações por Crotalaria spp., Solanum malacoxylon e Manihot spp. para cada planta $(0,96 \%$ dos surtos de intoxicação) foram registrados no LAP/FAMEZ. Com relação à Crotalaria spp., no Brasil sabe-se que, sob condições naturais, em intoxicações de animais de produção, a Crotalaria mucronata e C. juncea afetam o sistema respiratório e que a C. retusa, C. specatabilis e $C$. incana causam intoxicações que afetam principalmente o fígado (LEMOS et al., 1997; NOBRE et al., 2004; BOGHOSSIAN et al., 2007; UBIALI et al., 2011; TOKARNIA et al., 2012; QUEIROZ et al., 2013). Embora o surto tenha ocorrido em condição de pastoreio, não foi possível identificar a espécie da planta envolvida. Há um surto de intoxicação por C. mucronata com a predominância de lesões pulmonares, registrado no LAP/FAMEZ, porém as amostras foram encaminhadas do estado de Minas Gerais (LEMOS et al., 1997). Intoxicação por S. malacoxylon ocorreu no município de Aquidauana, no Pantanal Sul Mato-grossense, com sinais clínicos, achados de necropsia e histopatológico semelhantes aos descritos em estudos anteriores (PARDI; SANTOS, 1947; DÖBEREINER et al., 1971; TOKARNIA;
DÖBEREINER, 1974). No surto de intoxicação por Manihot spp., os casos ocorreram em uma propriedade leiteira, onde o proprietário relatou a ingestão de "mandioca brava" pelos animais. Variedades de Manihot spp., conhecidas como "brava", são mais rica em glicosídeo cianogênico responsável pelo quadro clínico-patológico observado (TOKARNIA et al., 2012). Os sinais clínicos do presente estudo corroboram relatos anteriores (AMORIM et al., 2004), não havendo lesões histológicas significativas (TOKARNIA et al., 2012).

Outras plantas são mencionadas por produtores e veterinários como causadoras de intoxicação, como o Stryphnodendron fissuratum e a Brachiaria radicans, entretanto, nenhum caso suspeito destas intoxicações foi confirmado pelo LAP/FAMEZ. Em relação ao $S$. fissuratum, surtos espontâneos foram descritos em Mato Grosso e a doença foi reproduzida experimentalmente com frutos colhidos em uma propriedade de Mato Grosso do Sul, na qual ocorreram mortes com quadro clínico-patológico compatível com a intoxicação, porém não foram realizados necropsias e exames histopatológicos nos casos espontâneos (FERREIRA et al., 2009).

\section{Outras doenças tóxicas}

De janeiro de 2000 a dezembro de 2012, aproximadamente $11,11 \%$ dos diagnósticos de doenças tóxicas realizados em bovinos foram atribuídas ao grupo outras doenças tóxicas. Dentre estas se destacam, em ordem decrescente, as intoxicações por chumbo (30,77\%), ureia $(23,08 \%)$, cloreto de sódio $(15,38 \%)$, abamectina $(15,38 \%)$ e os acidentes ofídicos (15,38\%).

Intoxicação por chumbo em bovinos nesse levantamento (quatro surtos) foi causada pela inalação de vapores de chumbo da fumaça produzida por uma usina de reciclagem de baterias de automóveis, que apresentou falhas no sistema de filtragem. A intoxicação por chumbo ocorre quando bovinos ingerem acidentalmente produtos, pastagens ou aguadas contaminadas e pela inalação de vapores 
(RADOSTITS et al., 2007). Em todos os casos acompanhados, não foram observadas alterações macroscópicas significativas, porém outros autores já descreveram alterações como edema cerebral (TRAVERSO et al., 2004), áreas focais de malácia e cavitações do córtex telencefálico, principalmente no lobo occipital (SUMMERS; CUMMINGS; LAHUNTA, 1995). Os achados histopatológicos foram semelhantes aos descritos por Traverso et al. (2004). Além das lesões histológicas sugestivas, o diagnóstico foi confirmado pelas concentrações de chumbo no fígado e rins de duas vacas, no solo e na grama onde os bovinos adoeceram. Este surto de intoxicação por chumbo em bovinos foi relatado pela equipe do LAP/FAMEZ e colaboradores (LEMOS et al., 2004).

Nos três surtos de intoxicação por ureia, registrados no LAP/FAMEZ, os bovinos foram encontrados mortos e, em um destes, foi constatada a morte dos animais cerca de 50 minutos após a administração de ureia misturada à palha de arroz e ao farelo. A evolução clínica superaguda que culmina com a morte dos bovinos em poucos minutos, é uma característica dessa intoxicação, sendo comum encontrar bovinos mortos próximos aos cochos (BARROS et al., 2006). Nestes surtos, não foi possível determinar a dose de ureia administrada aos animais, mas se sabe que doses altas $(0,5 \mathrm{~g} / \mathrm{kg}$ PV) de ureia granulada ou extrusada administradas experimentalmente a bovinos causaram intenso quadro tóxico em até 240 minutos após a ingestão da mesma (ANTONELLI, 2003). Em todos os casos do presente estudo, não foram encontradas alterações macro e microscópicas significativas, o que é uma característica da intoxicação (BARROS et al., 2006).

Os casos de intoxicação por cloreto de sódio (dois surtos) foram provenientes de materiais de bovinos encaminhados ao LAP/FAMEZ, os quais apresentavam sintomatologia nervosa, sem histórico de evolução clínica e com lesões histológicas que se caracterizavam por meningoencefalite eosinofílica, similar àquelas descritas em casos de intoxicação por sódio em suínos e que é considerada uma alteração típica da intoxicação por sal em suínos (SUMMERS; CUMMINGS; LAHUNTA, 1995; BOOS et al., 2012). Esta alteração ocorre na fase aguda, sendo que após 24 horas os eosinófilos são gradativamente substituídos por macrófagos, o que torna difícil o diagnóstico morfológico (CARLTON; MCGAVIN, 1995). Nos surtos descritos no presente estudo, não foi possível realizar a dosagem dos níveis de sódio no líquido cefalorraquidiano, exame confirmatório que tem sido descrito em intoxicações por sal/privação de água em suínos (BOSS et al., 2012) e também em bovinos (NAKAZATO; LEMOS; RIET-CORREA, 2000). Considerando que a meningoencefalite eosinofílica nem sempre é encontrada na intoxicação por sal em bovinos (VERDES et al., 2007), este não deve ser o único critério de diagnóstico, devendo se observar o histórico (intoxicação por sal/privação de água) e a dosagem de sódio.

Os acidentes ofídicos corresponderam a $15,38 \%$ (dois surtos) das outras doenças tóxicas diagnosticadas e são sugestivos de envenenamento botrópico, pois foram caracterizados por lesões necróticas nos locais de inoculação (TOKARNIA; PEIXOTO, 2006). Há poucos relatos de casos naturais de acidentes letais por Bothrops em bovinos no Brasil, entretanto, experimentos foram realizados com o veneno de serpentes desse gênero em bovinos, porém com pouca ou nenhuma descrição detalhada do quadro clínico-patológico (ARAÚJO; BELLUOMINI, 1962; ARAÚJO; ROSENFELD; BELLUOMINI, 1963; NOVAES et al., 1986).

Dois surtos de intoxicação por abamectina $(15,38 \%)$, utilizada em dosagem não ajustada de acordo com o peso do animal, foram observados em bovinos no município de Corumbá, com a recuperação de alguns animais. As alterações neurológicas observadas foram compatíveis com as manifestações relatadas em intoxicações descritas em outros estados brasileiros. É provável que a intoxicação por abamectina seja frequente e que o número de casos de intoxicação iatrogênica seja subestimado, visto que os sinais clínicos podem ser leves, transitórios e muitos animais apresentam rápida recuperação (SEIXAS et al., 2006). 


\section{Conclusões}

Dentre as causas tóxicas de mortalidade de bovinos em Mato Grosso do Sul, as plantas tóxicas são as mais importantes. As principais plantas causadoras de intoxicação foram Brachiaria spp., Vernonia rubricaulis, Amorimia pubiflora, Senna occidentalis e S. obtusifolia. Embora as plantas do gênero Brachiaria sejam responsáveis pelo maior percentual de intoxicação, sua importância como planta tóxica é pequena quando se relaciona o número de surtos com o consumo e a área ocupada por esta forrageira. Entretanto, o número de casos subnotificados pode alterar a importância da Brachiaria spp. como planta tóxica.

\section{Referências}

AMORIM, S. L.; MEDEIROS, R. M. T.; RIET-CORREA, F.; OLIVEIRA, A. C. P. Intoxicação experimental com plantas cianogênicas em bovinos. Pesquisa Veterinária Brasileira, Seropédica, v. 24, p. 5-6, 2004. Suplemento.

ANTONELLI, A. C. Administração de doses padrão e alta de uréia extrusada ou granulada em bovinos: uma análise clínica-toxicológica e laboratorial. 2003. Dissertação (Mestrado em Clínica Veterinária) Faculdade de Medicina Veterinária e Zootecnia, Universidade de São Paulo, São Paulo. Disponível em: $<$ http://www.teses.usp.br/teses/disponiveis/10/10136/ tde-25092007-134559/>. Acesso em: 9 fev. 2013.

ARAÚJO, P.; BELLUOMINI, H. E. Toxicidade de venenos ofídicos. I. Sensibilidade específica de animais domésticos e de laboratório. Memórias do Instituto Butantan, São Paulo, v. 30, p. 143-156, 1962.

ARAÚJO, P.; ROSENFELD, G.; BELLUOMINI, H. E. Toxicidade de venenos ofídicos. II. Doses mortais para bovinos. Arquivos do Instituto Biológico, São Paulo, v. 30, n. 8, p. 43-48, 1963.

ASSIS, T. S.; MEDEIROS, R. M. T.; RIET-CORREA, F.; GALIZA, G. J. N.; DANTAS, A. F. M.; OLIVEIRA, M. D. Intoxicações por plantas diagnosticadas em ruminantes e equinos e estimativa das perdas econômicas na Paraíba. Pesquisa Veterinária Brasileira, Seropédica, v. 30, n. 1, p. 13-20, 2010.

BARROS, C. S. L.; DRIEMEIER, D.; DUTRA, I. S.; LEMOS, R. A. A. Doenças do sistema nervoso de bovinos no Brasil. Montes Claros: Vallée, 2006. 207 p.
BARROS, C. S. L.; ILHA, M. R. S.; BEZERRA, J. R. O. S.; LANGOHR, I. M.; KOMMERS, G. D. Intoxicação por Senna occidentalis em bovinos em pastoreio (Leg. Caesalpinoideae). Pesquisa Veterinária Brasileira, Seropédica, v. 19, n. 2, p. 68-70, 1999.

BECKER, M.; CALDEIRA, F. H. B.; CARNEIRO, F. M.; OLIVEIRA, L. P.; TOKARNIA, C. H.; RIETCORREA, F.; LEE, S. T.; COLODEL, E. M. Importância da intoxicação por Amorimia pubiflora (Malpighiaceae) em bovinos em Mato Grosso: reprodução experimental da intoxicação em ovinos e bovinos. Pesquisa Veterinária Brasileira, Seropédica, v. 33, n. 9, p. 1049-1056, 2013.

BOGHOSSIAN, M. R.; PEIXOTO, P. V.; BRITO, M. F.; TOKARNIA, C. H. Aspectos clínico-patológicos da intoxicação experimental pelas sementes de Crotalaria mucronata (Fabaceae) em bovinos. Pesquisa Veterinária Brasileira, Seropédica, v. 27, n. 4, p. 149-156, 2007.

BOOS, G. S.; WATANABE, T. T. N.; ALMEIDA, P. R.; OLIVEIRA, L. G. S.; PAVARINI, S. P.; ZLOTOWSKI, P.; DRIEMEIER, D. Surto de intoxicação por sal em suínos em Santa Catarina. Pesquisa Veterinária Brasileira, Seropédica, v. 32, n. 4, p. 329-332, 2012.

BRASIL. Ministério do Planejamento, Orçamento e Gestão. Rio de Janeiro: Instituto Brasileiro de Geografia e Estatística, 2012. (Produção de Pecuária Municipal, v. 39). Disponível em: <http://ftp.ibge.gov.br/Producao Pecuaria/Producao_da_Pecuaria_Municipal/2011/ tabelas_pdf/tab03.pdf $\overline{\text { }}$. Acesso em: $\overline{5}$ dez. 2012.

BRUM, K. B.; HARAGUCHI, M.; LEMOS, R. A. A.; RIET-CORREA, F.; FIORAVANTE, M. C. Crystal associated cholangiopathy in sheep grazing Brachiaria decumbens containing the saponin protodioscin. Pesquisa Veterinária Brasileira, Seropédica, v. 27, n. 1, p. 39-42, 2007.

BRUM, K. B.; PURISCO, E.; LEMOS, R. A. A.; RIETCORREA, F. Intoxicação por Vernonia rubricaulis em bovinos no Mato Grosso do Sul. Pesquisa Veterinária Brasileira, Seropédica, v. 22, n. 3, p. 119-128, 2002.

CARLTON, W. W.; MCGAVIN, M. D. Thomson's special veterinary pathology. 2. ed. London: Mosby, 1995. 654 p.

CARMO, P. M. S.; IRIGOYEN, L. F.; LUCENA, R. B.; FIGHERA, R. A.; KOMMERS, G. D.; BARROS, C.S. L. Spontaneous coffee senna poisoning in cattle: Report on 16 outbreaks. Pesquisa Veterinária Brasileira, Seropédica, v. 31, n. 2, p. 139-46, 2011.

CARVALHO, N. M.; ALONSO, L. A.; CUNHA, T. G.; RAVEDUTTI, J.; BARROS, C. S. L.; LEMOS, R. A. A. Intoxicação de bovinos por Tetrapterys multiglandulosa (Malpighiaceae) em Mato Grosso do Sul. Pesquisa Veterinária Brasileira, Seropédica, v. 26, n. 3, p. 139146, 2006. 
CARVALHO, N. M.; BACHA, F. B.; SANTOS, A. C.; CARVAlHO, A. Q.; FACCIN, T. C.; POTT, A.; LEMOS R. A. A. Spontaneous and experimental intoxication of cattle by Simarouba versicolor A. St.-Hill (Simaroubaceae). Toxicon, Oxford, v. 64, p. 55-59, 2013.

CASTRO, M. B.; SANTOS, J. R. H. L.; MUSTAFA, V. S.; GRACINDO, C. V.; MOSCARDINI, A. C. R.; LOUVANDINI, H.; PALUDO, G. R.; BORGES, J. R. J.; HARAGUCHI, M.; FERREIRA, M. B.; RIETCORREA, F. Brachiaria spp. poisoning in sheep in Brazil: experimental and epidemiological findings. In: INTERNATIONAL SYMPOSIUM ON POISONOUS PLANTS, 8., 2009, João Pessoa. Anais... Cambridge: Cabi, 2009. p. 110-117.

CORRÊA, S. E.; VIEIRA, A.; COSTA, P. F.; CEZAR, M. I. Sistema semi-intensivo de produção de carne de bovinos Nelore no Centro-Oeste do Brasil. Campo Grande: Embrapa Gado de Corte, 2000. 5 p.

CRUZ, C.; DRIEMEIER, D.; PIRES, V. S.; COLODEL, E. M.; TAKETA, A. T. C.; SCHENKEL, E. P. Isolation of steroidal sapogen in simplicated in experimentally induced cholangiopathy of sheep grazing Brachiaria decumbens in Brazil. Veterinary Human Toxicology, Manhattan, v. 42, n. 3, p. 142-145, 2000.

CRUZ, C.; DRIEMEIER, D.; PIRES, V. S.; SCHENKEL, E. P. Experimentally induced cholangiopathy by dosing sheep with fractionate dextracts from Brachiaria decumbens. Journal Veterinary Diagnostic Investigation, Columbia, v. 13, n. 2, p. 170-172, 2001.

CRUZ, R. A. S.; OLIVEIRA, L. P.; CALDEIRA, F. H. B.; MENDONÇA, F. S.; BACHA, F. B.; POTT, A.; LEMOS, R. A. A.; COLODEL, E. M. Intoxicação espontânea e experimental por Pterodon emarginatus (Fabaceae Faboideae) em bovinos e experimental em ovinos. Pesquisa Veterinária Brasileira, Seropédica, v. 32, n. 11, p. 1087-1094, 2012.

DÖBEREINER, J.; TOKARNIA, C. H.; COSTA, J. B. D.; CAMPOS, J. L. E.; DAYRELL, M. S. "Espichamento", intoxicação de bovinos por Solanum malacoxylon, no Pantanal de Mato Grosso. Pesquisa Agropecuária Brasileira, Série Veterinária, Brasília, v. 6, p. 91-117, 1971.

EMPRESA BRASILEIRA DE PESQUISA AGROPECUÁRIA - EMBRAPA. Cultivo e uso do estilosantes-campo-grande. Campo Grande: Embrapa Gado de Corte, 2007. 11 p. (Comunicado técnico, 105).

Nota técnica. Uso correto do estilosantes em pastagens consorciadas. Campo Grande: Embrapa Gado de Corte, 2010. 3 p.
FERREIRA, J. L. M.; RIET-CORREA, F.; SCHILD, A. L.; MÉNDEZ, M. C. Intoxicação por Amaranthus spp (Amaranthaceae) em bovinos no Rio Grande do Sul. Pesquisa Veterinária Brasileira, Seropédica, v. 1, n. 3-4, p. 49-54, 1991.

FERREIRA, E. V.; BOABAID, F. M.; ARRUDA, L. P.; LEMOS, R. A. A.; SOUZA, M. A.; NAKAZATO, L.; COLODEL, E. M. Intoxicação por Stryphnodendron fissuratum (Mimosoideae) em bovinos. Pesquisa Veterinária Brasileira, Seropédica, v. 29, n. 11, p. 951957, 2009.

FERREIRA-NETO, P. G.; LEMOS, R. A. A.; MORI, A. E.; GUIMARÃES, E. B. Intoxicação por Senna occidentalis (Leg. Caesalpinoidea) em bovinos em pastoreio rotacionado. In: SIMPÓSIO LATINOAMERICANO DE PLANTAS TÓXICAS, PESQUISA VETERINÁRIA BRASILEIRA, 1., 2004, Salvador. Anais... Salvador: Colégio Brasileiro de Patologia Animal, 2004. v. 24, p. 22-23.

FIGHERA, R. A. Causas de morte e razões para eutanásia em cães. 2008. Tese (Doutorado em Medicina Veterinária) - Universidade Federal de Santa Maria. Centro de Ciências Rurais, Santa Maria.

GRECCO, F. B.; MANTAS, A. F. M.; RIET-CORREA, F.; LEITE, C. G. D.; RAPOSO, J. B. I. Cattle intoxication from Enterolobium contortisiliquum pods. Veterinary Human Toxicology, Manhattan, v. 44, n. 3, p. 160-162, 2002.

HENSON, J. B.; DOLLAHITE, J. W.; BRIDGES, C. H.; RAO, R. R. Myodegeneration in cattle grazing Cassia Species. Journal of the American Veterinary Medical Association, Chicago, v. 147, n. 2, p. 142-145, 1965.

JABOUR, F. F.; OLIVEIRA, L. I.; FRANÇA, T. N.; NOGUEIRA, V. A.; BRITO, M. F. Intoxicação por polpa cítrica em bovino. In: CONGRESSO BRASILEIRO DE BUIATRIA, 6., 2005, Búzios. Anais... Búzios: Associação Brasileira de Buiatria, 2005. p. 45.

LEMOS, R. A. A.; DUTRA, I. S.; SOUZA, G. F.; NAKAZATO, L.; BARROS, C. S. L. Intoxicação espontânea por Crotalaria mucronata em bovinos em Minas Gerais. Arquivos do Instituto Biológico, São Paulo, v. 64, p. 46, 1997. Suplemento.

LEMOS, R. A. A.; DRIEMEIER, D.; GUIMARÃES, E. B.; DUTRA, I. S.; MORI, A. E.; BARROS, C. S. L. Lead poisoning in cattle grazing pasture contaminated by industrial waste. Veterinary Human Toxicology, Manhattan, v. 46, n. 6, p. 326-328, 2004.

LEMOS, R. A. A; GUIMARÃES, E. B.; CARVALHO, N. M.; NOGUEIRA, A. P. A.; SANTOS, B. S.; SOUZA, R. I. C.; CARDINAL, S. G.; KASSAB, H. O. Plant 
poisonings in Mato Grosso do Sul. In: INTERNATIONAL SYMPOSIUM ON POISONOUS PLANTS, 8., 2009, Paraíba. Anais... Paraíba: CAB International, 2009. p. 68-72.

MCGAVIN, M. D.; ZACHARY, J. F. Bases da patologia em veterinária. 4. ed. Rio de Janeiro: Elsevier/Mosby, 2009. $1475 \mathrm{p}$.

MENDONÇA, F. S.; EVÊNCIO-NETO, J.; BARATELLA-EVÊNCIO, L.; DÓRIA, R. G. S.; FREITAS, S. H.; PELEGRINI, L. F.; CRUZ, R. A. S.; FERREIRA, E. V.; COLODEL, E. M. Natural and experimental poisoning of cattle by Enterolobium contortisiliquum Pods (Fabaceae Mimosoideae) in Central-Western Brazil. Acta Veterinaria Brno, Brno, v. 78, p. 621-625, 2009.

NAKAZATO, L.; LEMOS, R. A. A.; RIET-CORREA, F. Polioencefalomalacia em bovinos nos estados de Mato Grosso do Sul e São Paulo.Pesquisa Veterinária Brasileira, Seropédica, v. 20, n. 3, p. 119-125, 2000.

NOBRE, V. M. T.; RIET-CORREA, F.; BARBOSA FILHO, J. M.; DANTAS, A. F. M.; TABOSA, I. M.; VASCONCELOS, J. S. Intoxicação por Crotalaria retusa (Fabaceae) em equinos no semiárido da Paraíba. Pesquisa Veterinária Brasileira, Seropédica, v. 24, n. 3 , p. 132-143, 2004.

NOVAES, A. P.; LUCAS, S.; ABE, A. S.; FERNANDES, W.; PORTO, G.; ALMEIDA, I. L. Envenenamento botrópico em bovinos: tratamento opcional. São Carlos: Embrapa, UEPAE, 1986. 29 p. (Circular técnica, 3).

NUNES, S. G.; BOOK, A.; PENTEADO, M. I. O.; GOMES, D. T. Brachiaria brizantha cv. Marandu. 2. ed. Campo Grande: EMBRAPA-CNPGC, 1985. 28 p.

OSWEILLER, G. D.; BUCK, W. B.; BICKNELL, E. J. Production of perirenal edema in swine with Amaranthus retroflexus. American Journal Veterinary Research, Chicago, v. 30, n. 4, p. 557-566, 1969.

PARDI, M. C.; SANTOS, J. A. Ossificação pulmonar e calcificação vascular em bovinos do pantanal matogrossense. Veterinária, Rio de Janeiro, v. 1, n. 3, p. 3-7, 1947.

PAVARINI, S. P.; SOARES, M. P.; BANDARRA, P. M.; GOMES, D. C.; BANDINELLI, M. B.; CRUZ, C. E. F.; DRIEMEIER, D. Mortes súbitas em bovinos causadas por Amorimia exotropica (Malpighiaceae) no Rio Grande do Sul. Pesquisa Veterinária Brasileira, Seropédica, v. 31, n. 4, p. 291-296, 2011.

PEDROSO, P. M. O.; PESCADOR, C. A.; OLIVEIRA, E. C.; SONNE, L.; BANDARRA, P. M.; RAYMUNDO, D. L.; DRIEMEIER, D. Intoxicações naturais por plantas em ruminantes diagnosticadas no setor de Patologia
Veterinária da UFRGS no período de 1996-2005. Acta Scientiae Veterinariae, Porto Alegre, v. 35, n. 2, p. 213 218, 2007.

PEIXOTO, P. V.; BRUST, L. A. C.; BRITO, M. F.; FRANÇA, T. N.; CUNHA, B. R. M.; ANDRADE, G. B. Intoxicação natural por Amaranthus spinosus (Amaranthaceae) em ovinos no Sudeste do Brasil. Pesquisa Veterinária Brasileira, Seropédica, v. 23, n. 4, p. 179-184, 2003.

PIERCE, K. R.; O'HARA, P. J. Toxic myopathy in Texas cattle. South Western Veterinarian, v. 20, n. 2, p. 179184, 1967.

QUEIROZ, G. R.; RIBEIRO, R. C. L.; ROMÃO, F. T. N. M. A.; FLAIBAN, K. K. M. C.; BRACARENSE, A. P. F. R. L.; LISBÔA, J. A. N. Intoxicação espontânea de bovinos por Senna obtusifolia no estado do Paraná. Pesquisa Veterinária Brasileira, Seropédica, v. 32, n. 12, p. 1263-1271, 2012.

QUEIROZ, G. R.; RIBEIRO, R. C. L.; FLAIBAN, K. K. M. C.; BRACARENSE, A. P. F. R. L.; LISBÔA, J. A. N. Intoxicação espontânea por Crotalaria incana em bovinos no norte do estado do Paraná. Semina: Ciências Agrárias, Londrina, v. 34, n. 2, p. 823-832, 2013.

RADOSTITS, E. M.; GAY, C. C.; BLOOD, D. C.; HINCHCLIFF, K. W. Veterinary medicine. $10^{\text {th }}$ ed. London: Saunders, 2007. 2065 p.

RIET-CORREA, F.; MEDEIROS, R. M. T. Intoxicações por plantas em ruminantes no Brasil e no Uruguai: importância econômica, controle e riscos para a saúde pública. Pesquisa Veterinária Brasileira, Seropédica, v. 21, n. 1, p. 38-42, 2001.

RIET-CORREA, F.; MEDEIROS, R. M.; DANTAS, A. F. M. Plantas tóxicas da Paraíba. Patos: Universidade Federal de Campina Grande, 2006.

RIET-CORREA, G.; RIET-CORREA, F.; SCHILD, A. L.; DRIEMEIER, D. Wasting and death in cattle associated with chronic grazing of Brachiaria decumbens. Veterinary Human Toxicology, Manhattan, v. 44, n. 3, p. 179-180, 2002.

RISSI, D. R.; RECH, R. R.; PIEREZAN, F.; GABRIEL, A. L.; TROST, M. E.; BRUM, J. S.; KOMMERS, G. D.; BARROS, C. S. L. Intoxicações por plantas e micotoxinas associadas a plantas em bovinos no Rio Grande do Sul: 461 casos. Pesquisa Veterinária Brasileira, Seropédica, v. 27, n. 7, p. 261-268, 2007.

SANTOS, A. C.; CARVALHO, N. M.; RIBAS, N. L. K. S.; SANTOS, A. G. T.; LEAL, P. V.; LEMOS, R. A. A. Surtos de intoxicação por Senna occidentalis e Senna obtusifolia em bovinos a pastoreio. In: CONGRESSO BRASILEIRO DE BUIATRIA, 9., 2011, Botucatu. 
Anais... Botucatu: Faculdade de Medicina Veterinária e Zootecnia, 2011, p. 523-526.

SANTOS, A. C.; FACCIN, T. C.; CARVALHO, N. M.; LEAL, P. V.; POTT, A.; LEMOS, R. A. A. Intoxicação experimental por Simarouba versicolor (Simaroubaceae) em ovinos e indução de resistência ao consumo da planta. Pesquisa Veterinária Brasileira, Seropédica, v. 33, n. 3, p. 299-304, 2013.

SEIXAS, J. N.; PEIXOTO, P. V.; ARMIÉN, A. G.; JABOUR, F. F.; BRITO, M. F. Aspectos clínicos e patogenéticos da intoxicação por abamectina em bezerros. Pesquisa Veterinária Brasileira, Seropédica, v. 26, n. 3, p. 161-166, 2006.

SOUTO, M. A. A.; KOMMERS, G. D.; BARROS, C. S. L.; PIAZER, J. V. M.; RECH, R. R.; RIET-CORREA, F.; SCHILD, A. L. Neoplasias do trato alimentar superior de bovinos associadas ao consumo espontâneo de samambaia (Pteridium aquilinum). Pesquisa Veterinária Brasileira, Seropédica, v. 26, n. 2, p. 112-122, 2006 a.

SOUTO, M. A. M.; KOMMERS, G. D.; BARROS, C. S. L.; RECH, R. R.; PIAZER, J. V. M. Neoplasmas da bexiga associados à hematúria enzoótica bovina. Ciência Rural, Santa Maria, v. 36, n. 5, p. 1647-1650, 2006 b.

SOUZA, R. I. C.; RIET-CORREA, F.; BRUM, K. B.; FERNANDES, C. E.; BARBOSA-FERREIRA, M.; LEMOS, R. A. A. Intoxicação por Brachiaria spp em bovinos no Mato Grosso do Sul. Pesquisa Veterinária Brasileira, Seropédica, v. 30, n. 12, p. 1036-1042, 2010.

SPLENGLER, R. O.; BUSSINATI JÚNIOR, L. C.; NOGUEIRA, A. P. A.; LEMOS, R. A. A. Intoxicação por polpa cítrica em bovinos confinados em Mato Grosso do Sul.In:ENCONTRONACIONALDELABORATÓRIOS DE DIAGNÓSTICO VETERINÁRIO - ENDIVET, 2006, Campo Grande. Anais... Campo Grande: Equali, 2006. CD-ROM.

SUMMERS, B. A.; CUMMINGS, J. F.; LAHUNTA, A. Veterinary neuropathology. London: Mosby, 1995. 527 p.

TAKEUTI, K. L.; RAYMUNDO, D. L.; BANDARRA, P. M.; OLIVEIRA, L. G. S.; BOABAID, F. M.; BARRETO, L.; DRIEMEIER, D. Surto de intoxicação por Senna occidentalis em bovinos em pastoreio. Acta Scientiae Veterinariae, Porto Alegre, v. 39, n. 1, p. 954, 2011.

THRUSFIELD, M. Epidemiologia veterinária. 2. ed. São Paulo: Roca, 2004. 556 p.
TOKARNIA, C. H.; DÖBEREINER, J. "Espichamento", intoxicação de bovinos por Solanum malacoxylon, no Pantanal de Mato Grosso II. Estudos complementares. Pesquisa Agropecuária Brasileira, Série Veterinária, Brasília, v. 9, n. 7, p. 53-62, 1974.

Intoxicação de bovinos por Vernonia rubricaulis (Compositae) em Mato Grosso. Pesquisa Veterinária Brasileira, Seropédica, v. 2, n. 4, p. 143-147, 1982.

TOKARNIA, C. H.; PEIXOTO, P. V. A importância dos acidentes ofídicos como causa de mortes em bovinos no Brasil. Pesquisa Veterinária Brasileira, Seropédica, v. 26, n. 2, p. 55-68, 2006.

TOKARNIA, C. H.; BRITO, M. F.; BARBOSA, J. D.; PEIXOTO, P. V.; DÖBEREINER, J. Plantas tóxicas do Brasil. 2. ed. Rio de Janeiro: Helianthus, 2012. 566 p.

TRAVERSO, S. D.; LORETTI, A. P. L.; DONINI, M. A.; DRIEMEIER, D. Lead poisoning in cattle in Southern Brazil. Arquivo Brasileiro de Medicina Veterinária e Zootecnia, Belo Horizonte, v. 56, n. 3, p. 418-421, 2004.

UBIALI, D. G.; BOABAID, F. M.; BORGES, N. A.; CALDEIRA, F. H. B.; LODI, L. R. PESCADOR, C. A.; SOUZA, M.A.; COLODEL, E. M. Intoxicação aguda com sementes de Crotalaria spectabilis (Leg. Papilionoideae) em suínos. Pesquisa Veterinária Brasileira, Seropédica, v. 31, n. 4, p. 313-318, 2011.

UBIALI, D. G.; SILVA, R. G. F.; OLIVEIRA, L. P.; MORAES, L. G.; CALDEIRA, F. H. B.; PESCADOR, C. A.; COLODEL, E. M. Obstrução intestinal em bovinos causada pelo consumo de Stylosanthes ssp. (Fabaceae Papilionoideae). Pesquisa Veterinária Brasileira, Seropédica, v. 33, n. 2, p. 148-154, 2013.

VERDES, J. M.; MORAÑA, A.; RIET-CORREA, F.; GIMENO, E. J.; RIVERO, R.; DUTRA, F.; LEMOS, R. A. A.; EASTON, C.; MORAES, J.; ZONONIANI, R.; SANTOS, C. G.; CAPELLI, A.; DOMÍNGUEZ, R.; CALLIARI, A.; BATTES, D.; GUTIÉRREZ, F.; RUIZ, P. Neuropatología y neurotoxicología em ruminantes. Montevideo: Imprenta GEGA S. R. L., 2007. 145 p.

VIEIRA, R. N. F.; VARASCHIN, M. S.; BEZERRA JUNIOR, P. S.; WOUTERS, F.; LOURES, R. A.; NOGUEIRA, A. M.; PAVARINI, S. P. Intoxicação por polpa cítrica em bovinos de corte e de leite no Sul de Minas Gerais. In: ENCONTRO NACIONAL DE PATOLOGIA VETERINÁRIA, 13., 2007, Campo Grande. Anais... Campo Grande: Equali, 2007. CD-ROM. 